\title{
PIERO SRAFFA: UN ECONOMISTA SCONOSCIUTO?
}

\author{
Nota del m.e. PIER LUIGI PORTA (*)
}

(Adunanza del 17 marzo 2016)

SUNTO. - La nota delinea elementi essenziali per la ricostruzione dell'itinerario intellettuale di Piero Sraffa sulla base di documenti di archivio risalenti alla seconda metà degli anni venti del Novecento. Questi documenti attestano l'attenzione di Sraffa per il programma scientifico di Marx e insieme la sua insoddisfazione per l'insuccesso di Marx nel suo tentativo di collegare il proprio contributo teorico alla ricostruzione dei precedenti storici dello stesso. Le carte di archivio che documentano la fase formativa del programma di ricerca di Sraffa nella seconda metà degli anni venti mostrano l'intenzione di partire dalle premesse storiche per costruire sulla loro base una nuova teoria economica. Il successivo abbandono della trattazione storico-analitica conferma l'interpretazione di Luigi Pasinetti circa la vastità e ambizione del programma iniziale di Sraffa e la successiva delimitazione della sua ricerca entro confini realizzabili, di cui le 'equazioni' sono un aspetto caratteristico.

ABSTRACT. - This note outlines essential elements for reconstructing the intellectual route followed by Piero Sraffa on the basis of archival documents dating from the second half of the 1920s. These documents highlight Sraffa's interest in Marx's lack of success in linking his theoretical contribution to the reconstruction of its precedents in the history of political economy. Archival documents illustrate the formative phase of Sraffa's scientific programme and show his intention to build a new theory starting from its premises in the history of economic analysis. Sraffa's subsequent abandonment of the historical-analytical approach confirms Luigi Pasinetti's view that Sraffa had to shelve an initial programme of great vastity and ambition to reduce it within feasible limits, of which his 'equations' are a characteristic feature.

(*) Istituto Lombardo Accademia di Scienze e Lettere, Milano, Italy. E-mail: info@istitutolombardo.it 
Questa nota delinea gli elementi principali per una possibile plausibile ricostruzione del punto di vista iniziale di Piero Sraffa sull'economia politica classica in base a testimonianze fornite da documenti di archivio non pubblicati e risalenti alla seconda metà degli anni Venti.

Lo scopo di questa analisi è richiamare l'attenzione su specifici elementi di potenziale interesse per una discussione delle nuove prospettive di ricerca sul lavoro scientifico di Piero Sraffa. In particolare, attraverso la presente analisi sembra confermata l'interpretazione proposta da Luigi Pasinetti in scritti del 2001, 2007, 2012.

Il contributo interpretativo di Luigi Pasinetti è fondato su evidenza di archivio assai diretta e indiscutibile che Piero Sraffa, all'inizio del suo percorso di ricerca, avesse concepito un programma scientifico di fatto impossibile nella sua vastità e ambizione. Con il passare del tempo, Sraffa si trovò -gradualmente- costretto a circoscrivere entro confini realizzabili il suo programma (le sue "equazioni" dovrebbero essere lette come uno dei primi segni di questo più delimitato indirizzo di ricerca). Il compito principale (quello che Pasinetti chiama la revolution to be accomplished) finì così per essere affidato ad altri. L'idea fondamentale di questa interpretazione suggerisce insieme continuità e cambiamento nel percorso intellettuale di Sraffa. Questo è un punto di vista molto importante- un punto di vista proposto da Pasinetti anche in un suo recente libro (Pasinetti, 2007, 2010), libro che ha il merito di presentare una ricostruzione coerente e complessiva del percorso intellettuale di Sraffa. Questa ricostruzione, che è basata su documenti esistenti, ed è coerente con altri elementi di conoscenza del contesto, ha il carattere di una vera e propria biografia scientifica in nuce. L'argomentazione presentata nel mio saggio del 2012 (Porta, 2012) si basa, per metodo e contenuto, sulla linea di indagine seguita da Pasinetti. La differenza, rispetto all'analisi di Pasinetti, è che le iniziali concezioni Marxiane di Sraffa sono presentate in modo più esplicito nella ricostruzione biografica e scientifica, e che vi è un tentativo di esprimere con maggiore dettaglio la sostanza e i contenuti del programma di ricerca "impossibilmente ambizioso" seguito inizialmente da Sraffa.

Un punto specifico che occorre considerare è il significato del punto di partenza di Sraffa, a cui la maggior parte dei commentatori ha prestato insufficiente attenzione, con la rilevante eccezione di Pasinetti. Si tratta del dichiarato proposito di Sraffa di produrre un 
libro. Questo dichiarato proposito corrisponde alla dettagliata esposizione, nelle sue carte inedite, di un programma di ricerca ben definito.

Per brevità, ci possiamo qui riferire ad un singolo esempio, datato Novembre 1927, in cui si legge:

"Impostazione del libro.

L'unico sistema è di far la storia a ritroso, e cioè: stato attuale dell'economia; come vi si è giunti, mostrando le differenze e la superiorità delle vecchie teorie. Poi, esporre la teoria. /Se si va in ordine cronologico, Petty, Fisiocratici, Ricardo, Marx, Jevons Marshall, bisogna farlo precedere da uno statement della mia teoria per spiegare dove si "drive at": il che significa esporre prima tutta la teoria. E allora c'è il pericolo di finire come Marx, che ha pubblicato prima il Capitale, e poi non è riuscito a finite l'Histoire des Doctrines. E il peggio si è che non è riuscito a farsi capire, senza la spiegazione storica. /Il mio scopo è: I. esporre la storia, che è veramente l'essenziale/II. Farmi capire: per il che si richiede che io vada dal noto all'ignoto, da Marshall a Marx, dalla disutilità al costo materiale" (Cf. Sraffa papers, D3/12/11, item 55, novembre 1927).

L'iniziale ispirazione marxiana di Sraffa è evidente da un certo numero di documenti fra le carte di Sraffa, come quello in cui si trova il passo appena citato. In quello stesso torno di tempo, Sraffa dichiarò esplicitamente: "Prevedo che il risultato ultimo sarà una riformulazione di Marx" (si veda Porta, 2012, p. 1369). E' necessario prendere in considerazione il complesso di quei documenti se vogliamo discutere sostanza e contenuti del suo programma di ricerca "impossibilmente ambizioso", programma così opportunamente descritto nelle parole di Pasinetti.

Il punto di partenza nella costruzione del paradigma di Economia Classica secondo Sraffa è suggerito dal suo desiderio di seguire Marx e di fare meglio di Marx. E' un fatto che Sraffa, in particolare, rivolse allora particolare attenzione alle Teorie sul plusvalore. Più in generale, Sraffa mostrò particolare interesse al lato positivista del lavoro di Marx e della tradizione Marxiana (in quanto distinto dal lato utopico). D'altra parte, non ci dobbiamo stupire se questa complessiva ispirazione non si rivelò in modo esplicito nelle sue opere pubblicate, fatto che rende ancor più significativo lo studio dei manoscritti. Da un certo numero di note e appunti fra le carte di Sraffa, possiamo ipotizzare che 
Sraffa non ritenesse utile, in generale, discutere Marx in pubblico. Pasinetti osserva, nella sua ricostruzione della continuità e cambiamento in Sraffa, che molte questioni furono "trattate con grande circospezione, data la prevalente diffusa ostilità verso i punti di vista dei Classici e di Marx" (Pasinetti, 2001, p. 150; si veda anche Pasinetti, 2007, capitolo VI, p. 191).

Come abbiamo appena visto, in sede accademica, allora così come oggi, era difficile discutere in modo costruttivo il contributo economico di Marx. Al tempo stesso, lo slancio originale di Sraffa come economista (dopo i suoi iniziali contributi su moneta e finanza) trovò modo di esprimersi nei saggi da lui pubblicati sul sistema di Marshall. Di conseguenza, Marx non compare nei primi lavori pubblicati da Sraffa, benché sia indubbia l'ispirazione Marxiana per quello che egli chiamò privatamente (in appunti non pubblicati ma ben strutturati) il suo "Schema Generale".

In questa sede è opportuno aggiungere alcuni elementi di contesto, desunti da un'autorevole linea interpretativa proposta da Geoff Harcourt (1993) e di recente richiamati e confermati da Nerio Naldi in uno dei suoi scritti biografici su Sraffa. Piero Sraffa ricevette la sua prima educazione al Liceo Giuseppe Parini di Milano, dove uno dei suoi insegnanti, Domenico Re, gli trasmise l'interesse per gli ideali socialisti. "Più probabilmente, tuttavia, fu a Torino fra il 1912 e il 1916, con i suoi compagni al Liceo Massimo D'Azeglio, che Sraffa si avvicinò in modo più approfondito a temi economici e Marxiani in particolare". Molti dei suoi compagni erano Marxisti, ma i suoi insegnanti non avrebbero permesso una discussione esplicita in aula di Marx e di temi Marxisti. Da studente, Sraffa lesse anche i Principles di Ricardo e scoprì che molto di quello che Ricardo aveva da dire era molto simile a quello che egli aveva letto negli scritti di Marx. Dal momento che Ricardo era rispettato e accettabile dai suoi maestri, Sraffa e i suoi compagni presero a discutere temi Marxiani nella forma di uno studio di Ricardo (si veda Naldi, 2001, pp. 23-24). Il Professor Harcourt ha confermato, in una comunicazione personale del 16 settembre 2015, che questa interpretazione "è anche quello che Krishna Bharadwaj mi aveva detto, sulla base della sua lunga frequentazione con Piero Sraffa".

Sraffa era stato un intellettuale Marxiano completo fin dalla giovinezza, ed aveva un'enorme conoscenza dei diversi filoni della letteratura rilevante. Questo rende impossibile negare che i suoi interessi Marxiani fossero sin dall'inizio così ampi da confermare quello che 
Pasinetti descrive come "un grandioso programma di ricerca". Le iniziali scoperte di Sraffa non dovrebbero essere viste come eccessivamente interessate alle equazioni di prezzo di per sé: esse riguardano piuttosto i suoi tentativi di comprendere il sistema capitalistico. Non è difficile condividere la tesi di Giancarlo De Vivo (De Vivo, 2003, p. 6), secondo cui le equazioni di Sraffa sono un prodotto laterale della sua lettura o rilettura di Marx (De Vivo, 2003, p. 6) e che obiettivo di Sraffa per tutti gli anni Venti fu (come leggiamo nelle carte dello stesso Sraffa) quello di portare a compimento "una riformulazione di Marx...una traduzione di Marx in Inglese".

Una comprensione adeguata delle idee di Sraffa sull'economia classica richiede di tenere conto di due elementi storico-analitici:

(i) dal lato costruttivo, l'ispirazione tratta dalle Theorien über den Mehrwert di Marx,

e insieme

(ii) dal lato negativo e critico, l'esigenza profondamente sentita di contrastare la sintesi Marshalliana in economia.

Sraffa era profondamente convinto che una ricostruzione storico-analitica della teoria economica fosse un primo passo della massima importanza. Per quanto questa ricostruzione richiedesse un percorso assai complesso, il suo nucleo essenziale avrebbe potuto essere espresso in modo molto semplice, come Sraffa stesso sottolineò nella sua discussione su obiettivo e significato della teoria del valore nell'economia politica:

"Il concetto stesso di 'teoria del valore' ha subito una profonda trasformazione, a seconda del problema che in ciascun periodo ha maggiormente attratto l'attenzione degli economisti...

I. Cause e natura della ricchezza (1776-1820)

II. Distribuzione del prodotto fra le classi (1820-1870)

III. Determinazione del prezzo di singole merci.

Un aspetto rilevante in questo sviluppo è il continuo progresso dalla concezione filosofica e generale alla concezione tecnica e particolare. Questa tendenza è comune a tutte le scienze nel loro sviluppo...

Il problema pratico messo a fuoco nel primo periodo è 'come 
aumentare la ricchezza nazionale'; nel secondo periodo 'come cambiare la sua distribuzione, oppure come giustificare la distribuzione attuale'; nel terzo periodo 'come spiegare e come prevedere un cambiamento nel prezzo di un articolo'.

Due insiemi di cause hanno contribuito a produrre questo cambiamento. In primo luogo il progresso generale dell'economia come scienza, con il conseguente spostamento dalla considerazione di ampie questioni filosofiche all'analisi tecnica del meccanismo attraverso cui si raggiunge l'equilibrio economico. In secondo luogo, il cambiamento nelle questioni pratiche che debbono essere considerate dagli economisti; è interessante l'influenza di queste ultime su teorie che si suppongono astratte e senza alcuna applicazione pratica.

La teoria del valore-lavoro fu concepita da Ricardo come strumento per colpire i proprietari di terre... Ma successivamente, essendo stata adottata da Marx per colpire i capitalisti, fu necessario per i difensori del sistema attuale concepire una nuova teoria, la teoria del valore basata sull'utilità.

Nel caso di Ricardo, non si deve pensare che egli fosse parziale in modo consapevole nella sua teoria...

Nel caso di Marx, il fatto che la teoria del valore basata sull'utilità fosse stata prima formulata in più di un'occasione (da Dupuit e Gossen) e non avesse riscosso particolare attenzione, mentre essa trovò subito una larga opinione pronta ad accoglierla quando fu di nuovo presentata negli scritti pubblicati simultaneamente da Jevons, Menger e Walras in anni immediatamente successivi alla pubblicazione del vol. I del Capitale, è un fatto piuttosto significativo (Ashley, Present Position of Political Economy, EJ 1907).

[Da notare che lo sviluppo successivo ad opera di Marshall, che si ritenne essere altrettanto efficace nel rimuovere le basi della teoria del valore di Marx, non è affatto incompatibile con essa] (Sraffa papers D3/12/3, nn.9-11, estate 1927, parentesi quadre nel manoscritto originale)".

Questo è quello che Sraffa aveva in mente. Non emerge in superficie negli articoli pubblicati: è inteso che debba essere espresso nelle Lezioni all'Università di Cambridge, che a loro volta (nelle intenzioni di Sraffa) erano uno stadio preliminare rispetto al libro. La teoria del valore passa così in primo piano e si raccoglie la sfida di stabilire una rigorosa e "seria" teoria, costruita sulla base del concetto di "costo fisico reale”. Le Lezioni di Cambridge sono naturalmente un documento molto importante, ma in questa sede si è preferito mettere prima di tutto a fuoco una selezione di documenti dei tardi anni Venti, quindi 
scritti nel periodo in cui il pensiero di Sraffa sembra avere prodotto una serie di tentativi non pubblicati di stabilire un collegamento fra le sue critiche pubbliche del sistema Marshalliano (nei suoi noti articoli del 1925 e 1926) e il suo progetto privato di ricostruzione positiva dell'approccio classico alla teoria economica. La congettura che qui si presenta di un'ispirazione Marxiana di Sraffa come interprete degli economisti classici è interamente confermata dai documenti, che risultano essenziali per aggiungere un numero rilevante di aspetti e prospettive originali (Porta, 2012, in particolare paragrafo 3).

E' a questo punto che dobbiamo tornare alla tesi di Pasinetti su continuità e cambiamento (Pasinetti, 2001, 2007, 2012).

Nel caso di Sraffa, l'evoluzione (continuità e cambiamento) può essere descritta nel modo seguente. Il giovane Sraffa concepisce inizialmente un "programma di ricerca impossibilmente ambizioso" per dare vita ad un libro (si veda sopra, "Impostazione del libro") e ispirato a tre "linee di ragionamento" (Pasinetti, 2001, pp. 143-45), e cioè: 1) una condizione di stupore alla vista della "distorsione aberrante" che "aveva avuto luogo nella teoria economica nella seconda parte del diciannovesimo secolo"; 2) la necessità urgente "di sviluppare una critica impietosa delle aberrazioni prodotte dalla teoria economica marginale" (corsivo aggiunto) rispetto a temi quali distribuzione, valore, utilità, interesse, $\mathrm{e}$ così via); e 3 ) "come conseguenza logica", "il ritorno al punto dove si trovava una sensata teoria economica" attraverso a) "l'eliminazione di tutte le difficoltà" che avevano afflitto gli economisti classici e Marx e b) lo sviluppo della "teoria economica rilevante e vera così come avrebbe dovuto evolversi a partire da Petty, Cantillon, i Fisiocratici, Smith, Ricardo, Marx".

Il documento più importante di questa fase iniziale sono (come abbiamo già indicato) le Lectures on Advanced Theory of Value. Già alla fine degli anni Trenta, tuttavia, Sraffa si era reso conto della "completa impossibilità di dare concretamente forma ad un programma di ricerca così tremendamente ambizioso" (Pasinetti, 2001, p. 140) Per fortuna, gli era stato permesso di "porre termine all'incubo di fare lezione". Gli fu così possibile prendere in mano il progetto di edizione degli scritti di Ricardo, che definì la seconda fase della sua evoluzione intellettuale: "il suo principale grandioso programma di ricerca fu messo temporaneamente da parte". Sraffa vi si dedica nuovamente all'inizio degli anni Quaranta, quando "la parte principale degli scritti di Ricardo era stata inviata alle stampe". A questo punto Sraffa ritorna "al suo programma e 
comincia a dare forma ad una nuova fase che, dalle sue note, sembra portarlo a concentrarsi sulla formulazione corretta, in termini di equazioni di almeno alcune fra le sue "proposizioni Classiche". In questo modo, "l'orizzonte del suo programma di ricerca è drasticamente ridotto".

Questa è la corretta interpretazione delle equazioni di Sraffa.

La conclusione tratta da Pasinetti, sulla base dell'analisi qui presa in esame, è illuminante. Pasinetti si chiede "Quale frazione del programma originario è stata portata a compimento?". La risposta è che "la prima e la seconda linea di ricerca nel programma originario di Sraffa -due linee della massima importanza stando alle sue note- erano state di fatto abbandonate". In particolare, ciò che sorprende è che l'abbandono della prima linea di ricerca porti a trascurare del tutto la trattazione strico-analitica, che come abbiamo visto era del tutto centrale nell'originario progetto di ricerca di Sraffa. "Appare quasi incredibile -scrive Pasinetti- che dopo avere rimproverato Marx ... per non avere presentato prima una spiegazione storica, ed avere sostenuto che questa omissione era stata la causa dei fraintendimenti di cui era stato vittima, abbia poi finito per fare lo stesso".

Possiamo in effetti essere stupiti, perché -possiamo chiederciripetere gli stessi errori?

Pasinetti ha descritto in modo efficace quello che possiamo definire un senso di frustrazione. Ludwig Wittgenstein- il cui sodalizio intellettuale con Sraffa è oggetto di ricerche- usava dire a molti dei suoi amici che le discussioni con Sraffa lo lasciavano con la sensazione di un albero dal quale fossero stati tagliati tutti i rami. Lo stesso destino attende gli studiosi di Sraffa: e Pasinetti ha reso in modo efficace questa sensazione (si veda anche Sen, 2003, in particolare, p. 1242).

\section{RIFERIMENTI BIBLIOGRAFICI}

Ashley, W.J. (1907) 'The Present Position of Political Economy', The Economic Journal, 17 (68), dicembre, pp. 467-89.

de Vivo, G. (2003) 'Sraffa's Path to Production of Commodities by Means of Commodities. An Interpretation', Contributions to Political Economy, 22 (1, novembre), pp. 1-25.

Harcourt, G.C. (1993) 'Cambridge Economic Tradition”, in J.E. King (a cura di), The Elgar Companion to Post Keynesian Economics, Cheltenham, Glos., UK, Northampton, Mass., USA: Edward Elgar Publishing Limited, 2003, pp. 44-51.

Naldi, N. (2001) 'Piero Sraffa's Early Approach to Political Economy: from the Gymnasium to the Beginning of his Academic Career', in T. Cozzi e R. 
Marchionatti (a cura di), Piero Sraffa's Political Economy - A Centenary Estimate, London, Routledge, pp. 23-40.

Pasinetti, L.L. (2001) 'Continuity and Change in Sraffa's Thought-An Archival Excursus', in T. Cozzi e R. Marchionatti (a cura di), Piero Sraffa's Political Economy - A Centenary Estimate, London, Routledge, pp. 139-56.

Pasinetti, L.L. (2007) Keynes and the Cambridge Keynesians: A Revolution in Economics' to be Accomplished, Cambridge, UK, Cambridge University Press.

Pasinetti, L.L. (2010) Keynes e i Keynesiani di Cambridge. Una 'rivoluzione in economia' da portare a compimento, Roma - Bari, Laterza.

Pasinetti, L.L. (2012) 'Piero Sraffa and the Future of Economics', Cambridge Journal of Economics, 36 (6, novembre), pp. 1303-14.

Porta, P.L. (2012) 'Piero Sraffa's Early Views on Classical Political Economy', Cambridge Journal of Economics, 36 (6, novembre), pp. 1357-83.

Sen, A.K. (2003) 'Sraffa, Wittgenstein, and Gramsci”, Journal of Economic Literature, 41 (4, dicembre), pp. 1240-55. 
